\title{
LAYOUT OF AN ELECTROSTATIC STORAGE RING AT IAP*
}

\author{
Carsten P. Welsch, Alwin Schempp, Johann Wolfgang Goethe Universität \\ Institut für Angewandte Physik \\ Frankfurt am Main, Germany
}

\section{Abstract}

The design of a small electrostatic storage ring for all different kinds of ions at energies up to $50 \mathrm{keV}$ is presented in this paper.

After a general introduction to this field, the different electrostatic elements are presented. The requirements of the vacuum system, field quality and adjustment accuracies are given. Finally, the present status is described.

\section{PROJECT DESCRIPTION}

\subsection{General}

During the last decades, one focus of research at IAP was the design and construction of heavy ion linacs especially RFQs and spiral loaded cavities. A wide range of experiments can be covered with these machines.

In order to extend possibilities to the fields of decay processes, beam interaction and storage of large biomolecules, it has been decided to design a small electrostatic storage ring for ions with energies up to 50 $\mathrm{keV}[1]$.

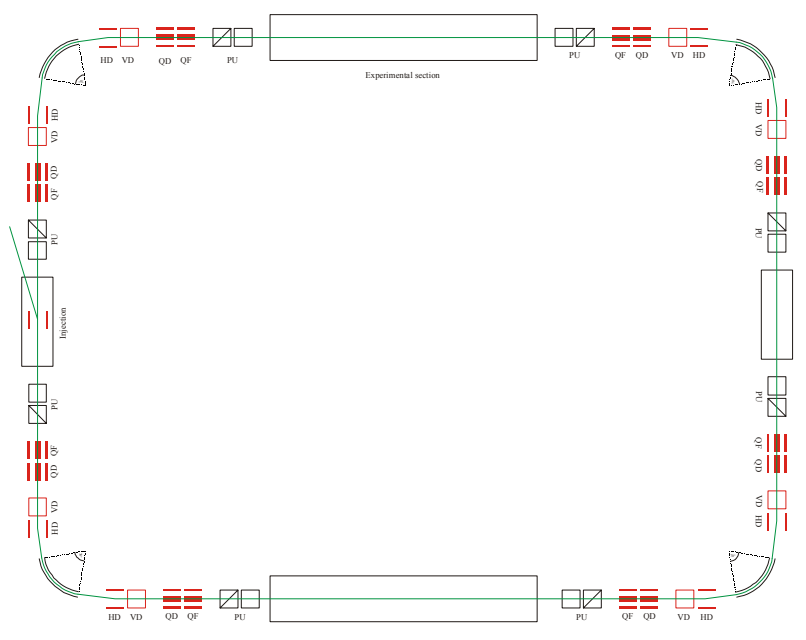

Figure 1: Layout of the complete ring

Such a device may be seen as a cross between an electromagnetic trap and "classical" rings.
Low costs, small size combined with energy independence of the necessary fields and good accessibility of the experimental sections are some of the interesting properties. Possible experiments with such a machine cover a wide range: Especially the advantage of being able to store heavy biomolecules for an extended period of time and the absence of magnetic fields makes experiments possible one cannot cover with magnetic rings.

In principle, the geometrical shape of an electrostatic machine is not limited by any rules. However, beam energy, aperture and required space for later experiments do reduce the number of possible layouts to a few.

\subsection{Beam properties}

The beam will leave the ECR ion source [2] with an energy of up to $50 \mathrm{keV}$, corresponding to a velocity of roughly $0.01 \mathrm{c}$. Thus relativistic effects can be neglected in all calculations.

The aperture defines the maximum size of the circulating beam and is $50 \mathrm{~mm}$ in the present design.

One main difference to magnetic type rings is the massindependence of the necessary fields. Theoretically, one can therefore set up the fields for one charge/energy relation and switch from one ion type to another without changing any of the rings' parameters.

This advantage could already be verified at the two existing electrostatic rings at ISA [3] and in Tsukuba [4].

\section{LATTICE}

\subsection{Injection}

The beam will enter the ring at an angle of $10^{\circ}$ and is bend towards the axis by an electric "fast kicker" inflector of two $100 \mathrm{~mm}$ × $70 \mathrm{~mm}$ copper plates placed at a difference of $50 \mathrm{~mm}$.

The field of $1.8 \mathrm{kV} / \mathrm{cm}$ is switched off after the beam has traveled half the circumference of the machine, i.e. $1.5 \mu \sec (\mathrm{p})$.

\footnotetext{
*Work supported by BMBF

${ }^{\dagger}$ c.welsch@iap.uni-frankfurt.de
} 


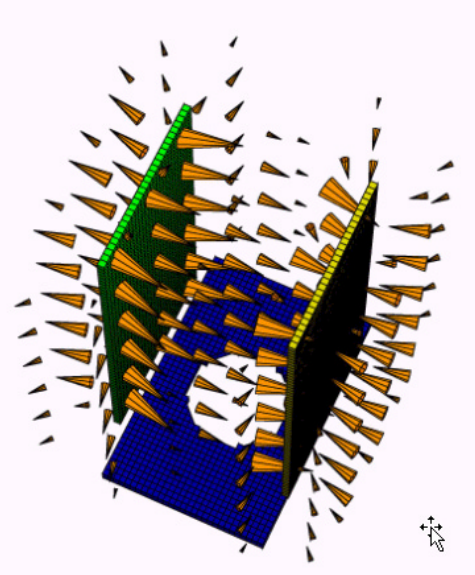

Figure 2: MAFIA plot of the calculated field in a $10^{\circ}$ electrostatic deflector

\subsection{Bending sections}

The bending is done in two steps: First, a $10^{\circ}$ electrostatic deflector bends the beam away from the straight section, afterwards a $70^{\circ}$ cylindrical deflector and another $10^{\circ}$ parallel plate deflector add up to a $90^{\circ}$ bend.

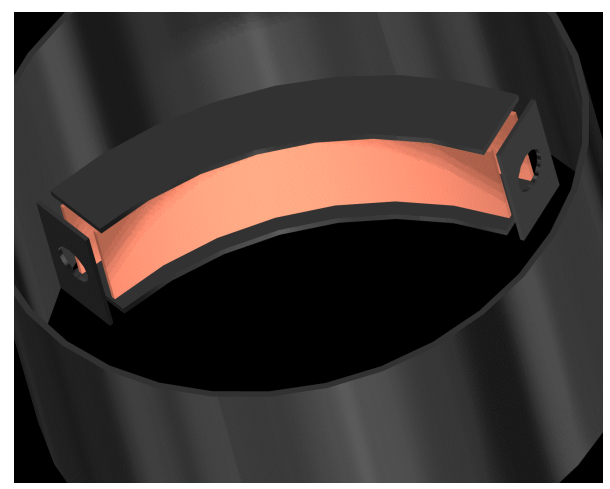

Figure 3: $70^{\circ}$ cylindrical deflector

This allows to detect neutral particles at the end of the straight sections. In addition interaction processes with laser or electron beams can be studied.

The cylindrical deflector will be placed in a pillbox cavity, which guarantees good accessibility and space for diagnostic elements. Its two plates are $3 \mathrm{~cm}$ apart, requiring a field of $4 \mathrm{kV} / \mathrm{cm}$ to keep the beam on a mean radius of $25 \mathrm{~cm}$. Zero Volt shields at the entrance and exit of the deflector limit the maximum size of the beam to 30 $\mathrm{mm}$ and reduce fringe fields.

It should be noted that, in contrast to magnetic bending sections, the longitudinal energy is not conserved. Particles entering the cylindrical deflector off-axis do see a longitudinal field component and therefore get accelerated or decelerated.

Finally, there is a stronger focusing in the horizontal plane as compared to magnetic bends, but no focusing in the vertical plane.

\subsection{Focusing elements}

Transverse modification of the circulating beam is done with pairs of quadrupole doublets. Electrodes of $10 \mathrm{~cm}$ length produce a field homogeneous enough to allow an aperture of about $5 \mathrm{~cm}$.

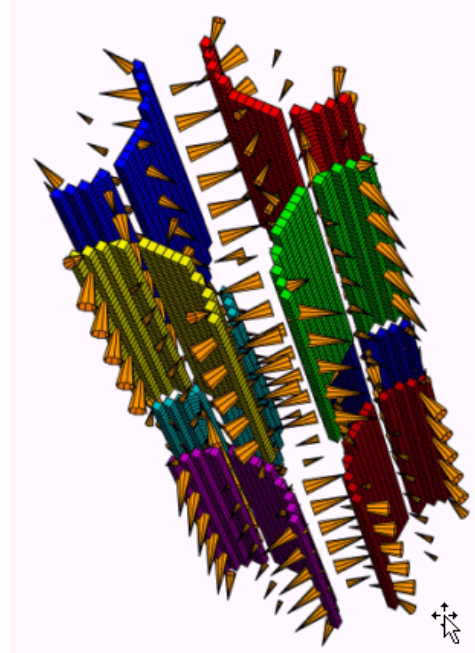

Figure 4: MAFIA plot of the quadrupole field

A total number of at least 4 doublets will give the possibility to change the properties of the beam in a wide range. The required voltages are up to $+/-1 \mathrm{kV}$, depending on the desired shape of the beam.

The ideal shape of hyperbolic electrode surfaces is approximated by cylindrical electrodes of radius $r=1.1468 \cdot r_{\text {aperture }}$, which is no problem at these low field levels.

\section{VACUUM SYSTEM}

The lifetime of the circulating beam is mainly limited by collisions with the residual gas and therefore a vacuum pressure in the order of $10^{-12}$ mbar should be envisaged. Therefore, all vacuum chambers will be built from stainless steel. Oxygen-free copper was chosen for the electrodes and Aluminum-Oxide for insulation purposes.

The whole system, including diagnostics and control system is bakable to $200^{\circ} \mathrm{C}$.

\section{DIAGNOSTICS}

Diagnostics will include beam-position monitors, Faraday cups, scrapers, CCD cameras and single particle detectors. Since only about $10^{5}$ ions will circulate the ring, a good signal / noise ratio of the amplifiers is needed [5].

The whole machine will be controlled by a LabView / Group3 system. 


\section{PRESENT STATUS AND OUTLOOK}

The present parameters of the planned storage ring are given in table 1 .

Table 1: List of design parameters

\section{General Parameters}

Maximum energy

$50 \mathrm{keV}$

Circumference

$10.71 \mathrm{~m}$

Revolution time

$3.5 \mu \mathrm{s}(\mathrm{p})$

$10^{\circ}$ deflectors

Plate area

Plate distance

Voltage

$$
\begin{array}{r}
100 \mathrm{~mm} \times 70 \mathrm{~mm} \\
50 \mathrm{~mm} \\
+/-4.5 \mathrm{kV}
\end{array}
$$

$70^{\circ}$ deflectors

Height

Radii

Voltage

$$
\begin{array}{r}
235 \mathrm{~mm} \text { and } 265 \mathrm{~mm} \\
+/-6 \mathrm{kV}
\end{array}
$$

$70 \mathrm{~mm}$

Quadrupoles

Length

Outer Radius of electrodes

Voltage

$100 \mathrm{~mm}$

$29 \mathrm{~mm}$

$+/-1 \mathrm{kV}$

The construction of a small pillbox cavity has just been completed. Its size has been chosen to be big enough to house all the different electrodes and diagnostic elements.

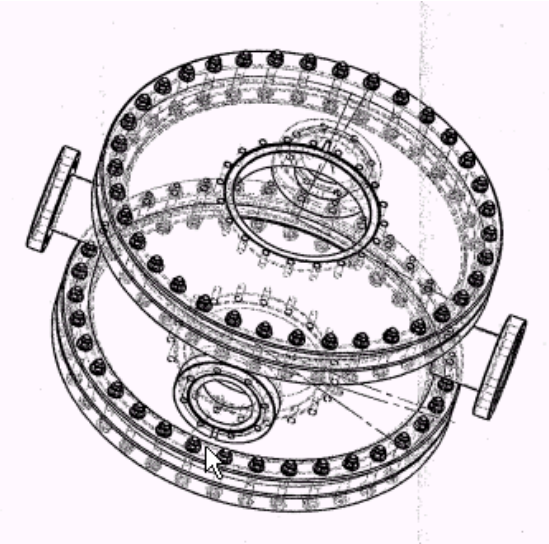

Figure 5: drawing of pillbox-type test cavity

The effects of electrode displacements, surface errors and fringe fields will be studied as well as questions arising when dealing with XHV. Furthermore, the control system will be tested.

The next step will be the construction of one quarter of the ring. This will allow to study injection, optimize the optical elements and integrate the diagnostic and control system.

\section{ACKNOWLEDGEMENTS}

The authors would like to thank Søren Pape Møller for his support throughout the design process.

This work was supported by BMBF and Studienstiftung des deutschen Volkes.

\section{REFERENCES}

[1] C.P. Welsch, "An electrostatic storage ring at IAP" Graduiertenkolleg, Annual Report

Frankfurt, Darmstadt, Germany, 2000

[2] H. Schmidt-Böcking et al, "The Frankfurt ECR-RFQ facility"

Rev. Sci. Instrum. 63 (4), 1992

[3] S.P. Møller, "ELISA, an electrostatic storage ring for atomic physics"

NIM A 394, 281 (1997)

[4] T. Tanabe, "An electrostatic storage ring for molecular science"

EPAC, Vienna, Austria, 2000

[5]L. Søby et al, “The Closed Orbit Measurement System for the CERN Antiproton Decelerator" DIPAC99, Daresbury Lab, UK 